\title{
Assessment of lithographic process variation effects in InGaAsP annular Bragg resonator lasers
}

\author{
William M. J. Green \\ Department of Electrical Engineering, California Institute of Technology, 1200 East California Boulevard, \\ Pasadena, California 91125 \\ Jacob Scheuer \\ Department of Applied Physics, California Institute of Technology, 1200 East California Boulevard, \\ Pasadena, California 91125
}

Guy A. DeRose, ${ }^{\text {a) }}$ Amnon Yariv, and Axel Scherer

Department of Electrical Engineering and Department of Applied Physics, California Institute of Technology, 1200 East California Boulevard, Pasadena, California 91125

(Received 4 June 2004; accepted 30 August 2004; published 10 December 2004)

\begin{abstract}
Optical microresonators based on an annular geometry of radial Bragg reflectors have been designed and fabricated by electron-beam lithography, reactive ion etching, and an epitaxial transfer process. Unlike conventional ring resonators that are based on total internal reflection of light, the annular structure described here is designed to support optical modes with very small azimuthal propagation coefficient and correspondingly large free spectral range. The effect of lithographic process variation upon device performance is studied. Laser emission wavelength and threshold optical pump power are found to vary between similar devices given different electron doses during electron-beam lithography. As the resonance wavelength and quality factor of these resonators are very sensitive to environmental changes, these resonators make ideal active light sources that can be integrated into large arrays for gas and liquid sensing applications and are easily interrogated. (C) 2004 American Vacuum Society. [DOI: 10.1116/1.1808740]
\end{abstract}

\section{INTRODUCTION}

The combination of high-resolution patterning and dry etching techniques have recently enabled the fabrication of lithographically defined optical structures for the efficient confinement of light. As the accuracy of pattern definition has evolved to below $100 \mathrm{~nm}$ linewidths, microfabricated periodic structures can be constructed within III-V semiconductor gain materials with sufficient accuracy to enable lithographic tuning of the spectral response of lasers. ${ }^{1}$ Thus, a new class of devices utilizing the high refractive index contrast present in air-clad semiconductor optical structures has emerged, and has been studied for the efficient localization of light in small volumes. The mode volume of a laser source is particularly important in environmental sensing applications and single-molecule spectroscopy, where it is desirable to probe as small a volume as possible. ${ }^{2}$ By combining advances in lithographic capabilities with high quality crystal growth, short Fabry-Perot cavity lasers, ${ }^{3}$ photonic crystal lasers, ${ }^{4}$ and folded cavity sources ${ }^{5}$ have recently been demonstrated. Here we describe a lithographically defined ring microcavity, based on optimally designed cylindrical Bragg reflectors surrounding a radial defect. ${ }^{6,7}$ Resonators of this class, known as annular Bragg resonators (ABRs), are designed to support azimuthally propagating modes, with energy concen trated within the defect region by radial Bragg reflection. Losses in these lasers are determined by the qual-

${ }^{\text {a) }}$ Author to whom correspondence should be addressed; electronic mail: derose@caltech.edu ity of the lithographic and dry etching procedures, as well as vertical diffraction losses that must be minimized through appropriate cavity design. ${ }^{8}$ Annular Bragg resonators are compact, demonstrate large free spectral ranges, and can be used to achieve efficient light interaction with small volumes of analyte and the integration of laser arrays with predetermined emission wavelengths within a small chip area. These attributes are ideally suited for on-chip spectroscopy and the identification of chemical and biological materials. The basic geometry of these devices is shown in Fig. 1, which depicts the internal and external gratings surrounding an annular defect. Here we will describe the fabrication of these devices, and focus on the influence of lithographic procedures on the performance of ABR lasers.

\section{DEVICE FABRICATION}

Annular Bragg resonators with large index contrast Bragg reflectors were realized in a membrane of active InGaAsP semiconductor material. The semiconductor medium consisted of a 250-nm-thick InGaAsP membrane, in which six strained InGaAsP quantum wells and their barrier layers (7.5 $\mathrm{nm}$ wells, $1 \%$ compressive strain; $12 \mathrm{~nm}$ barriers, $0.5 \%$ tensile strain, $\left.\lambda_{g}=1.2 \mu \mathrm{m}\right)$ were sandwiched between two 60.5 $\mathrm{nm}$ InGaAsP $\left(\lambda_{g}=1.1 \mu \mathrm{m}\right)$ layers. The peak photoluminescence (PL) from the quantum wells occurred at $\lambda_{\mathrm{PL}}$ $=1.559 \mu \mathrm{m}$. Epitaxial layers were grown by metalorganic chemical vapor deposition on an InP substrate.

The ABR fabrication process, illustrated in Fig. 2, pro-

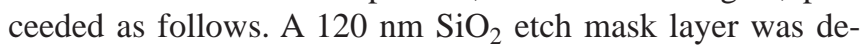




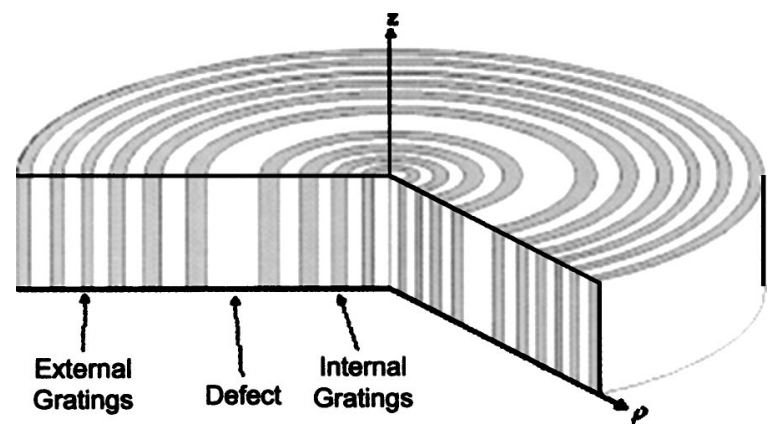

FIG. 1. Illustration of the annular defect mode resonator structure. Cylindrical Bragg gratings provide radial confinement of light propagating within the central defect region.

posited by plasma-enhanced chemical vapor deposition. Approximately $550 \mathrm{~nm}$ of polymethylmethacrylate (PMMA) electron-beam resist was then applied by spin-coating and baked. Several ABR geometries were patterned into the PMMA layer using a Leica Microsystems EBPG 5000+ direct electron-beam writer. The EBPG 5000+ system operated at $100 \mathrm{kV}$ accelerating voltage and $10 \mathrm{nA}$ beam current. Patterns were written with exposure doses of $625,650,675$, and $700 \mu \mathrm{C} / \mathrm{cm}^{2}$. Development of the patterned PMMA film was carried out in a solution of 1:3 MIBK:IPA for $60 \mathrm{~s}$. The scanning electron microscope (SEM) cross section through a typical PMMA pattern shown in Fig. 3(a) illustrates the results from this high-resolution lithography.

Subsequently, the PMMA patterns were transferred into the $\mathrm{SiO}_{2}$ etch mask layer by inductively coupled plasma reactive ion etching (ICP-RIE) using $\mathrm{C}_{4} \mathrm{~F}_{8}$ plasma, in an Oxford Instruments Plasmalab System100 tool. The remaining PMMA was removed with a gentle $\mathrm{O}_{2}$ plasma etching step.

a) Deposit SiO2 mask
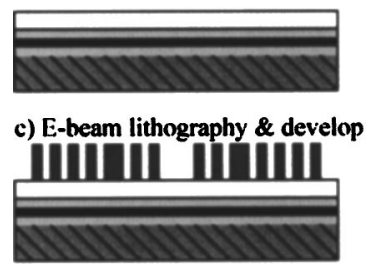

e) Strip PMMA mask and InGaAsP/InP ICP etch

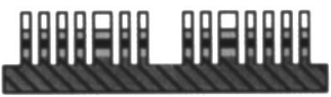

g) InGaAsP membrane release
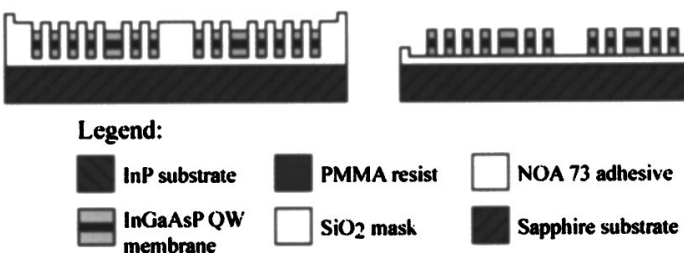

FIG. 2. Flow diagram for annular Bragg resonator fabrication and polymer bonding process. The dark region in the middle of the InGaAsP quantum well membrane is intended to represent the location of the quantum wells.

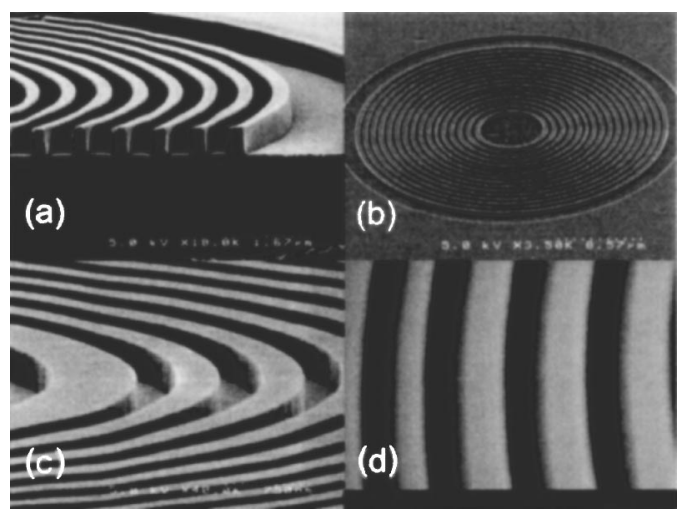

FIG. 3. SEM images of an annular Bragg resonator in various stages of the fabrication process. (a) Cross section of PMMA pattern after electron-beam lithography and development. The $\mathrm{SiO}_{2}$ mask layer can be seen between the PMMA and the substrate. The sample was coated with a thin layer of gold to prevent charging during imaging. (b) Image taken after HI/Ar ICP-RIE etch and $\mathrm{SiO}_{2}$ mask removal. The radial defect is the (slightly narrower) 6th ring from the center. (c) Magnified image of etched semiconductor grating, illustrating vertical and smooth sidewalls produced by HI/Ar etch process. (d) Magnified environmental SEM image of semiconductor rings, taken after epitaxial transfer to sapphire substrate and optical adhesive etch. Brighter regions are the top and side surfaces of the semiconductor rings, dark regions are the trenches from which the adhesive was removed.

The patterned $\mathrm{SiO}_{2}$ layer then served as a hard mask for transfer into the active InGaAsP membrane, using a lowvoltage ICP-RIE etch employing HI/Ar chemistry. ${ }^{9}$ The $\mathrm{HI} / \mathrm{Ar}$ system has two significant advantages over alternative chemical systems for etching InP-based semiconductors. First, no polymeric deposits form on the sample or chamber walls during etching, as typical with the $\mathrm{CH}_{4} / \mathrm{H}_{2} / \mathrm{Ar}$ gas chemistry. ${ }^{10}$ Furthermore, the etch products are sufficiently volatile at room temperature, and no sample heating is required to enhance material desorption, as is generally needed for $\mathrm{Cl}_{2} / \mathrm{Ar}$ reactive gas mixtures. ${ }^{11}$ In the etch recipe used, the HI/Ar gas flow was 10/6 sccm, the chamber pressure was $5 \mathrm{~m}$ Torr, and the ICP and rf electrodes were driven with $950 \mathrm{~W}$ and $30 \mathrm{~W}$, respectively. The plasma dc self-bias was $\sim 100 \mathrm{~V}$, and the etch rate was $\sim 325 \mathrm{~nm} / \mathrm{min}$. The $\mathrm{SiO}_{2}$-masked ABR samples were etched for $1 \mathrm{~min}$, to a depth completely through the InGaAsP membrane and partially into the InP substrate. The remaining $\mathrm{SiO}_{2}$ hard mask was then stripped in a buffered hydrofluoric acid solution. Several SEM images of a typical ABR device at this stage in the fabrication process are shown in Figs. 3(b) and 3(c). Fig. 3(b) provides a view of the entire resonator, whereas Fig. 3(c) illustrates the smooth anisotropic features of the HI/Ar etch used to define the Bragg reflectors.

To achieve strong out-of-plane optical confinement, the InGaAsP membrane must be clad by low-index material both above and below. An epitaxial layer transfer technique, ${ }^{12}$ using UV-curable Norland optical adhesive NOA $73(n \approx 1.56$ at $\lambda=1.55 \mu \mathrm{m}$ ), was adopted to facilitate transfer of the InGaAsP membrane to a transparent sapphire substrate. The optical adhesive was applied to the patterned InGaAsP surface by spin-coating, and the entire sample was flip-bonded to the sapphire substrate. The NOA 73 adhesive was then 
cured by illumination with an ultraviolet lamp through the sapphire. Subsequently, the InP substrate was removed by a combination of mechanical polishing and selective wet chemical etching, leaving only the 250 -nm-thick patterned active InGaAsP membrane encapsulated within the cured optical adhesive film. The transferred ABR devices demonstrated excellent adhesion over the entire sample. Finally, the adhesive filling the trenches was removed with an isotropic $\mathrm{NF}_{3} / \mathrm{O}_{2}$ ICP-RIE etch. The SEM image in Fig. 3(d) shows a magnified view of several transferred annular rings after adhesive removal. Comparison of Fig. 3(c) with Fig. 3(d) shows no roughening or milling of the semiconductor surfaces, suggesting that exposure to the $\mathrm{NF}_{3} / \mathrm{O}_{2}$ plasma process is not expected to generate any additional sources of optical scattering loss.

It is worthwhile to note that the choice of a low-index transfer substrate is not critical to the success of the polymer bonding technique employed here. The low-index NOA 73 adhesive layer is thick enough to optically isolate the ABR devices, and as such, common substrates such as a Si wafer or glass slide would function equally well as sapphire. Indeed, the ability to epitaxially transfer ABR devices in single units or large arrays to any substrate provides desirable flexibility for integration and application of ABR devices as key elements within chemical and biological sensing systems.

\section{EXPERIMENT}

Several resonators of the design shown in Fig. 3(b), fabricated using a range of electron exposure doses as discussed above, were tested to assess the effects of lithographic process variation. In this design, the Bragg reflectors were of mixed order, with second-order high-index and first-order low-index layers, and were designed for reflection at a center wavelength $\lambda=1550 \mathrm{~nm}$. While Bragg reflectors in Cartesian coordinates require gratings with a constant pitch, ${ }^{13}$ it has been shown that the optimal cylindrical Bragg reflector requires a "chirped" grating, in which the grating pitch changes as a function of radius. ${ }^{6}$ In the tested design, the grating pitch was nominally chirped from 0.91 to $0.81 \mu \mathrm{m}$ from the inner to the outer perimeter. The width of the highindex semiconductor defect was nominally $0.28 \mu \mathrm{m}$, chosen for a first-order defect, and the defect radius was $7.72 \mu \mathrm{m}$. The Bragg reflectors were composed of five periods to the inside of the radial defect, and 10 periods to the outside. For detailed discussion on the design of annular Bragg resonators and optimal cylindrical Bragg reflectors, the reader is directed to Refs. 6 and 7.

The ABR devices were pumped by pulsed optical excitation, using a mode-locked Ti:sapphire laser emitting $\sim 100 \mathrm{fs}$ full width at half-maximum pulses at a repetition rate of 77 $\mathrm{MHz}$, with a center wavelength of $\lambda_{p}=890 \mathrm{~nm}$. The pump beam was incident normal to the plane of the ABR devices, and focused through the transparent sapphire substrate with a $50 \times[$ numerical aperture $(\mathrm{NA})=0.42]$ microscope objective . Owing to the mixed-order design, the radial component of a wave resonant with the grating completes a full optical cycle between successive grating "periods." Thus, light diffracted

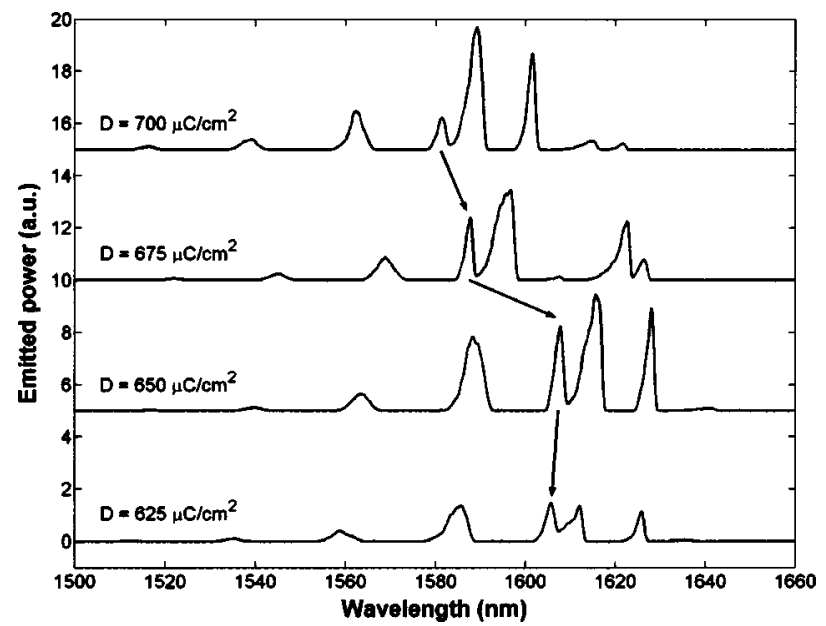

FIG. 4. Emission spectra from four different ABR lasers fabricated with varying electron dose $D$, illustrating effects of lithographic process variations on lasing mode wavelength. Arrows follow the redshift of a particular pair of optical modes resulting from the increasing semiconductor filling fraction with decreasing electron dose, for $D=700,675$, and $650 \mu \mathrm{C} / \mathrm{cm}^{2}$. The slight blueshift observed for the $D=625 \mu \mathrm{C} / \mathrm{cm}^{2}$ spectrum departs from this trend. Optical pump power was $1.4 \mathrm{~mW}$ for all spectra.

vertically from consecutive periods has phase differences of $2 \pi$, and therefore interferes constructively. ${ }^{14}$ The vertically emitted PL signal was collected from the side opposite to the pump, using a $50 \times(\mathrm{NA}=0.42)$ microscope objective. The collected PL was then focused into a multimode optical fiber and fed into an optical spectrum analyzer. Measurements were performed at room temperature.

Near infrared spectra obtained from four ABR lasers given different electron beam doses $D$ during lithography are shown in Fig. 4. The pump objective lens was defocused such that the pump spot diameter was $\sim 16 \mu \mathrm{m}$, in order to ensure reasonable overlap between the pump and the ABR defect. The time averaged pump power incident on each device was $1.4 \mathrm{~mW}$. While devices having the same dose exhibit almost identical spectra, comparison of the spectra from devices with $D=700,675$, and $650 \mu \mathrm{C} / \mathrm{cm}^{2}$ shows successive shifts of the lasing modes to longer wavelengths for decreasing electron dose. The position of a pair of closely spaced modes in each spectrum is used to track the redshift, as indicated by the arrows. The wavelengths of these modes are $1581 \mathrm{~nm} / 1589 \mathrm{~nm}$ for $D$ $=700 \mu \mathrm{C} / \mathrm{cm}^{2}, 1588 \mathrm{~nm} / 1596 \mathrm{~nm}$ for $D=675 \mu \mathrm{C} / \mathrm{cm}^{2}$, and $1608 \mathrm{~nm} / 1615 \mathrm{~nm}$ for $D=650 \mu \mathrm{C} / \mathrm{cm}^{2}$. The observed redshifts were approximately $7 \mathrm{~nm}$ between $D=700$ and $675 \mu \mathrm{C} / \mathrm{cm}^{2}$, and $20 \mathrm{~nm}$ between $D=675$ and $650 \mu \mathrm{C} / \mathrm{cm}^{2}$. As the exposure dose is decreased, the width of the PMMA rings remaining after development of the pattern [Fig. 3(a)] will increase, ultimately resulting in wider semiconductor rings in the Bragg reflectors and ABR defect. The resulting increase in the semiconductor filling fraction raises the effective index $n_{\text {eff }}$ of the guided optical modes, which in turn causes a redshift. The percent change in $n_{\text {eff }}$ is estimated to be $1.8 \%$ between $D=700$ and $675 \mu \mathrm{C} / \mathrm{cm}^{2}$, and $2.6 \%$ between $D=675$ and $650 \mu \mathrm{C} / \mathrm{cm}^{2}$. In contrast, the spectrum 


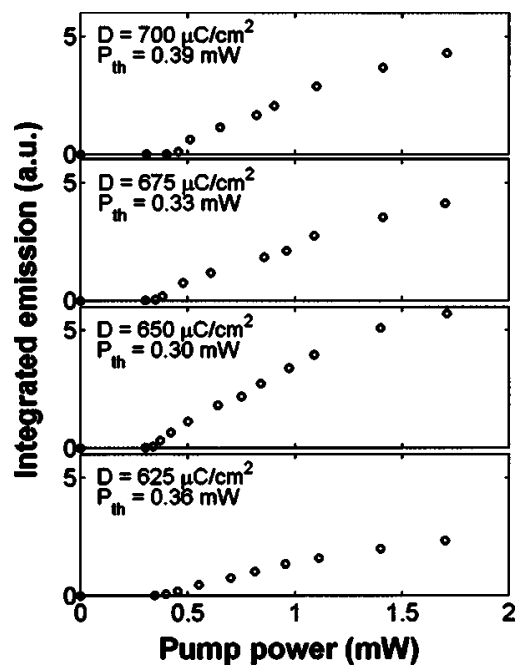

FIG. 5. $L-L$ plots from four ABR lasers fabricated with varying electron dose $D$. The increase in the modal gain with decreasing dose leads to successively decreasing threshold pump powers $P_{\text {th }}$ for $D=700 \mu, 675 \mu$, and $650 \mu \mathrm{C} / \mathrm{cm}^{2}$. The higher threshold pump power and poor efficiency of the $D=625 \mu \mathrm{C} / \mathrm{cm}^{2}$ device departs from this trend.

from the device patterned with $D=625 \mu \mathrm{C} / \mathrm{cm}^{2}$, in which the two closely spaced modes are located at 1605 and 1612 $\mathrm{nm}$, appears to be slightly blueshifted with respect to the $D$ $=650 \mu \mathrm{C} / \mathrm{cm}^{2}$ spectrum. This departure from the trend observed for larger electron doses is likely due to some additional nonsystematic source of fabrication process drift.

Plots of the integrated ABR emission versus optical pump power for each of the four devices are given in Fig. 5. As the electron dose is decreased, a decrease in the pump power $P_{\text {th }}$ required to reach laser threshold is observed. Thresholds occurred at $P_{\mathrm{th}}=0.39,0.33$, and $0.3 \mathrm{~mW}$ for $D=700,675$, and $650 \mu \mathrm{C} / \mathrm{cm}^{2}$, respectively. The increased semiconductor filling fraction also results in a larger overlap of the ABR modes with the amplifying quantum wells. The corresponding increase in the optical gain experienced by the ABR modes gives rise to a reduction in threshold pump power. Again, the $L-L$ curve for the device patterned with $D=625 \mu \mathrm{C} / \mathrm{cm}^{2}$ departs from the observed trend, demonstrating a larger threshold pump power of $0.36 \mathrm{~mW}$, and also having poor slope efficiency above threshold compared with devices given a larger dose. The higher value of $P_{\mathrm{th}}$ and the degraded efficiency lend further evidence for the degradation of this device by a nonsystematic fabrication error.

Although the IR near-field pattern emitted by the ABR is circularly symmetric, the lasing modes exhibit different polarization characteristics. Particularly, some of the modes are circularly polarized while the others are slightly elliptically polarized. We attribute the slight deviation of the polarization state of the modes from the expected circular polarization to fabrication errors that broke the radial symmetry of the device.

\section{CONCLUSIONS}

Lithographic techniques, originally developed for the microelectronics industry, have now been applied towards the spectral tuning and control of photonic devices, such as multiwavelength light sources. The fabrication tolerances obtained during the construction of such devices must be carefully controlled when constructing chip-based optical spectroscopy systems for biological and chemical detection, particularly when the detection scheme is based on resonant excitation of bonds within organic molecules. Here we have described a semiconductor laser source ideally suitable for the detection of small changes in the modal effective index or $Q$ factor, which can be integrated with a variety of potential substrates.

In summary, we have designed, fabricated, and characterized a compact optical cavity that supports lasing modes with a large free spectral range. Our cavity can easily be integrated with other photonic devices such as photonic crystals and distributed feedback lasers, within a single thin membrane of III-V semiconductor material. We have developed dry etching procedures based on $\mathrm{HI} / \mathrm{Ar}$ mixtures to precisely control the cavity geometries in such InGaAsP slabs. Roomtemperature lasing action from localized defect modes of the annular Bragg resonators was observed. The dependence of the laser wavelength and threshold upon electron exposure dose illustrates the need for careful control of the fabrication process, but also demonstrates that the $Q$ factor of the resonator's optical modes are reasonably tolerant to process variation. Our calculations predict that these cavities will also work well within high refractive index ambient environments as are needed for many chemical analysis systems.

\section{ACKNOWLEDGMENTS}

The authors would like to acknowledge generous support by the NSF, the AFOSR, and DARPA. Dr. Oskar Painter is also thanked for providing help with $\mathrm{SiO}_{2}$ ICP-RIE etch processing.

${ }^{1}$ O. Painter, A. Husain, A. Scherer, P. T. Lee, I. Kim, J. D. O'Brien, and P. D. Dapkus, IEEE Photonics Technol. Lett. 12, 1126 (2000).

${ }^{2}$ M. Loncar, A. Scherer, and Y. Qiu, Appl. Phys. Lett. 82, 4648 (2003).

${ }^{3}$ T. F. Krauss, O. Painter, A. Scherer, J. S. Roberts, and R. M. De La Rue, Opt. Eng. 37, 1143 (1998).

${ }^{4}$ M. Loncar, M. Hochberg, and A. Scherer, Opt. Lett. 29, 721 (2004).

${ }^{5}$ N. C. Frateschi, P. D. Dapkus, S. S. Ou, J. J. Yang, and M. Jansen, IEEE Photonics Technol. Lett. 5, 741 (1993).

${ }^{6}$ J. Scheuer and A. Yariv, J. Opt. Soc. Am. B 20, 2285 (2003).

${ }^{7}$ J. Scheuer and A. Yariv, IEEE J. Quantum Electron. 39, 1555 (2003).

${ }^{8}$ K. Srinivasan and O. Painter, Opt. Express 10, 670 (2002).

${ }^{9}$ S. J. Pearton, U. K. Chakrabarti, A. Katz, F. Ren, and T. R. Fullowan, Appl. Phys. Lett. 60, 838 (1992).

${ }^{10}$ U. Niggebrugge, M. Klug, and G. Garus, Inst. Phys. Conf. Ser. 79, 367 (1985).

${ }^{11}$ V. M. Donnelly, D. L. Flamm, C. W. Tu, and D. E. Ibbotson, J. Electrochem. Soc. 129, 2533 (1982).

${ }^{12}$ S. R. Sakamoto, C. Ozturk, Y. T. Byun, J. Ko, and N. Dagli, IEEE Photonics Technol. Lett. 10, 985 (1998).

${ }^{13}$ A. Yariv, Optical Electronics in Modern Communications, 5th ed. (Oxford University Press, New York, 1997).

${ }^{14}$ A. Shaw, B. Roycroft, J. Hegarty, D. Labilloy, H. Benisty, C. Weisbuch, T. F. Krauss, C. J. M. Smith, R. Stanley, R. Houdre, and U. Oestrle, Appl. Phys. Lett. 75, 3051 (1999). 